\title{
Limits on spin-dependent WIMP-nucleon cross-sections from the first ZEPLIN-II data
}

\author{
ZEPLIN-II Collaboration
}

\begin{abstract}
G.J. Alner ${ }^{\mathrm{a}}$, H.M. Araújo ${ }^{\mathrm{b}, \mathrm{a}}$, A. Bewick ${ }^{\mathrm{b}}$, C. Bungau ${ }^{\mathrm{a}, \mathrm{b}}$, B. Camanzi ${ }^{\mathrm{a}}$, M.J. Carson ${ }^{\mathrm{c}}$, R.J. Cashmore ${ }^{\text {d }}$, H. Chagani ${ }^{\text {c }}$, V. Chepel $^{\mathrm{e}}$, D. Cline $^{\mathrm{f}}$, D. Davidge ${ }^{\mathrm{b}}$, J.C. Davies ${ }^{\mathrm{c}}$, E. Daw ${ }^{\mathrm{c}}$,

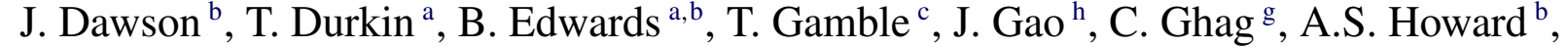
W.G. Jones ${ }^{\text {b }}$, M. Joshi ${ }^{\text {b }}$, E.V. Korolkova ${ }^{g}$, V.A. Kudryavtsev ${ }^{\text {c,* }}$, T. Lawson ${ }^{c}$, V.N. Lebedenko ${ }^{\text {b }}$, J.D. Lewin ${ }^{a}$, P. Lightfoot ${ }^{\mathrm{c}}$, A. Lindote ${ }^{\mathrm{e}}$, I. Liubarsky ${ }^{\mathrm{b}}$, M.I. Lopes ${ }^{\mathrm{e}}$, R. Lüscher ${ }^{\mathrm{a}}$, P. Majewski ${ }^{\mathrm{c}}$, K. Mavrokoridis ${ }^{c}$, J.E. McMillan ${ }^{c}$, B. Morgan ${ }^{c}$, D. Muna ${ }^{c}$, A.St.J. Murphy ${ }^{g}$, F. Neves ${ }^{\mathrm{e}}$, G.G. Nicklin ${ }^{\mathrm{c}}$, W. Ooi ${ }^{\mathrm{f}}$, S.M. Paling ${ }^{\mathrm{c}}$, J. Pinto da Cunha ${ }^{\mathrm{e}}$, S.J.S. Plank ${ }^{\mathrm{g}}$, R.M. Preece ${ }^{\mathrm{a}}$, J.J. Quenby ${ }^{\mathrm{b}}$, M. Robinson ${ }^{\mathrm{c}}$, G. Salinas ${ }^{\mathrm{h}}$, F. Sergiampietri ${ }^{\mathrm{f}, 1}$, C. Silva ${ }^{\mathrm{e}}$, V.N. Solovov ${ }^{\mathrm{e}}$, N.J.T. Smith ${ }^{a}$, P.F. Smith ${ }^{\text {a,f }}$, N.J.C. Spooner ${ }^{\text {c }}$, T.J. Sumner ${ }^{\text {b }}$, C. Thorne ${ }^{b}$, D.R. Tovey ${ }^{\text {c }}$, E. Tziaferi ${ }^{c}$, R.J. Walker ${ }^{\text {b }}$ H. Wang ${ }^{\text {f }}$, J. White ${ }^{\text {h }}$, F.L.H. Wolfs ${ }^{\text {i }}$

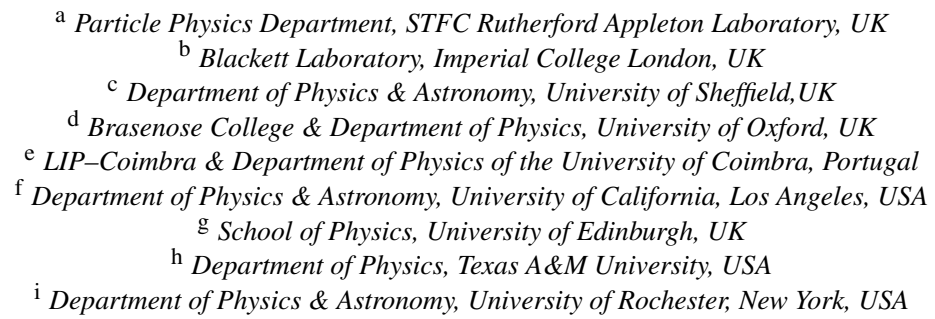

a Particle Physics Department, STFC Rutherford Appleton Laboratory, UK

b Blackett Laboratory, Imperial College London, UK

${ }^{\mathrm{c}}$ Department of Physics \& Astronomy, University of Sheffield,UK

${ }^{\mathrm{d}}$ Brasenose College \& Department of Physics, University of Oxford, UK

${ }^{\mathrm{e}}$ LIP-Coimbra \& Department of Physics of the University of Coimbra, Portugal

${ }^{\mathrm{f}}$ Department of Physics \& Astronomy, University of California, Los Angeles, USA $\mathrm{g}$ School of Physics, University of Edinburgh, UK

${ }^{\mathrm{h}}$ Department of Physics, Texas A\&M University, USA

${ }^{i}$ Department of Physics \& Astronomy, University of Rochester, New York, USA

Received 1 May 2007; received in revised form 20 July 2007; accepted 10 August 2007

Available online 15 August 2007

Editor: L. Rolandi

\begin{abstract}
The first underground data run of the ZEPLIN-II experiment has set a limit on the nuclear recoil rate in the two-phase xenon detector for direct dark matter searches. In this Letter the results from this run are converted into the limits on spin-dependent WIMP-proton and WIMP-neutron cross-sections. The minimum of the curve for WIMP-neutron cross-section corresponds to $7 \times 10^{-2} \mathrm{pb}$ at a WIMP mass of around 65 GeV. (c) 2007 Elsevier B.V. All rights reserved.
\end{abstract}

PACS: 95.35.+d; 14.80.Ly; 29.40.Mc; 29.40.Gx

Keywords: ZEPLIN-II; Dark matter; WIMPs; Liquid xenon; Radiation detectors

\section{Introduction}

* Corresponding author.

E-mail address: v.kudryavtsev@ sheffield.ac.uk (V.A. Kudryavtsev).

1 Also at INFN Pisa, Italy.
Weakly Interacting Massive Particles (WIMPs) remain the most plausible candidate for dark matter, responsible for about 
$80 \%$ of the total matter and for about $23 \%$ of the total energy contents of the Universe. WIMPs are expected to interact with ordinary matter via spin-independent (sometimes also called scalar or coherent) and spin-dependent (axial) interactions. Spin-independent interactions of WIMPs should largely dominate in high- $A$ targets due to the $A^{2}$ coherence enhancement factor (here $A$ is the atomic weight of the material used as a target). However, the relative probability of the spinindependent and spin-dependent interactions depends also on the particle content of WIMPs, i.e., on parameters of a particular supersymmetric model. In some models, WIMPs interact predominantly through the spin-dependent interactions. This stimulates the search for spin-dependent interactions of WIMPs in addition to the spin-independent case.

The interest in spin-dependent WIMP-nucleon interactions is enhanced by the positive WIMP signal claimed by the DAMA Collaboration. Although the first DAMA publications favoured spin-independent signal due to the very soft spectrum of events observed, further studies allowed the interpretation of the results in terms of the combination of both spin-independent and spin-dependent interactions [1].

The spin-dependent WIMP-nucleus cross-section depends on the spin factor of the nucleus that is primarily determined by the number of protons and neutrons in the nucleus, namely whether it is odd or even. For odd-proton nuclei the spindependent WIMP-nucleus cross-section is mainly due to the WIMP-proton interactions, whereas for odd-neutron nuclei it is dominated by WIMP-neutron scattering. For even-even nuclei the spin-dependent cross-section is highly suppressed.

Among the most sensitive targets for spin-dependent WIMP searches are $\mathrm{Na}, \mathrm{I}, \mathrm{Cs}, \mathrm{F}$ (all odd-proton) and $\mathrm{Xe}, \mathrm{Ge}$ (having odd-neutron isotopes). The best limits from direct detection experiments so far have been set by the NAIAD (WIMPproton) [2] and CDMS [3], ZEPLIN-I [4] and EDELWEISS [5] (WIMP-neutron) experiments.

In this Letter, the recent results of the ZEPLIN-II experiment are analysed in terms of the spin-dependent limits. A brief description of the detector and experimental data is given in Section 2. The method of calculating spin-dependent limits is presented in Section 3 together with the results. Conclusions are given in Section 4.

\section{ZEPLIN-II experiment}

ZEPLIN-II [6,7] is a two-phase (liquid/gas) xenon detector searching for elastic scattering of WIMPs off xenon nuclei. ZEPLIN-II is operated at the Boulby Underground Laboratory in the UK at a depth of $2805 \mathrm{~m}$ w.e. underground with a muon flux of $(4.09 \pm 0.15) \times 10^{-8}$ muons $/ \mathrm{cm}^{2} / \mathrm{s}$ [8]. The detector, data acquisition system, analysis procedure and experimental data are described in detail in Refs. [9-11]. We present here only a short summary of the detector performance and some other features important for further analysis.

ZEPLIN-II consists of a vacuum cryostat containing about $31 \mathrm{~kg}$ of liquid xenon. The target volume is viewed from above by seven $13 \mathrm{~cm}$ diameter photomultiplier tubes (PMTs). The inner surface of the xenon vessel is covered by light-reflecting PTFE for better collection of scintillation photons.

The detector records scintillation light and ionisation electrons from charged particles. Scintillation light is detected by the PMTs promptly after excitation of the active medium. Ionisation electrons are drifted through the liquid towards the surface by means of applied electric field, extracted from the liquid into the gas where they are accelerated producing a secondary scintillation signal as electroluminescence. The depth of liquid xenon $(14 \mathrm{~cm})$ corresponds to a maximum drift time of $73 \mu \mathrm{s}$ for electrons at the drift field of $1 \mathrm{kV} / \mathrm{cm}$.

To protect the target volume from radioactivity in rock (gammas and neutrons), the ZEPLIN-II detector is surrounded by hydrocarbon material and high-purity lead. Part of the hydrocarbon shielding is instrumented liquid scintillator that also acts as an anticoincidence system (active veto) preventing the signals, detected simultaneously in the target and in the veto, being interpreted as WIMP interactions.

The trigger is provided by five-fold coincidences between different PMTs at a single photoelectron level. Either a primary, $\mathrm{S} 1$, or a secondary, S2, signal can trigger the data acquisition. The signals from all seven PMTs are recorded with 2 ns sampling time during $\pm 100 \mu$ s around the trigger pulse, covering all possible arrival times for both primary and secondary signals. In an off-line data analysis three-fold coincidences between different PMTs at a single photoelectron level were used to identify and parameterise the primary signal $\mathrm{S} 1$.

A number of parameters have been measured for each waveform. They are listed and discussed in Refs. [9,10], the most important being the total areas of the primary and secondary pulses (proportional to the number of photoelectrons), S1 and $\mathrm{S} 2$, respectively, the time delay between them and the width of the pulses (that determines whether the pulse is the primary or secondary signal).

Position sensitivity of the experiment in the vertical direction is achieved by considering the time delay of the secondary pulse which is proportional to the drift time of the electrons and thus determines the point of the interaction along the drift field direction. This allows us to exclude events originating on or close to the grid wires that provide the electric field and are contaminated mainly with radon progeny. In the horizontal plane the event position is reconstructed using the relative pulse areas from secondary signals on different PMTs. This method gives bigger uncertainty compared to the drift time due to the large PMT sizes and small photon statistics at low energies.

A daily energy calibration of the detector using a ${ }^{57} \mathrm{Co}$ gamma-ray source allowed monitoring the stability of detector operation. The WIMP/gamma discrimination performance of ZEPLIN-II has been tested by calibrating the detector using high-energy gamma-ray $\left({ }^{60} \mathrm{Co}\right)$ and neutron $(\mathrm{AmBe})$ sources. High-energy gamma-rays produce the main electron recoil background, whereas fast neutrons scatter elastically off nuclei producing nuclear recoils in the same way as expected from WIMP scatters. Using the S2/S1 versus S1 plot from neutron calibration run (Fig. 8 in Ref. [9]) the nuclear recoil acceptance box has been defined as retaining $50 \%$ of nuclear recoil events at any given energy chosen for analysis. This acceptance box 
was expected to have a small number of electron recoils due to the tail of S2/S1 distribution observed in the gamma calibration run.

The first data run of the ZEPLIN-II detector had a live time of 31.2 days after time periods with unstable operation conditions were removed from the analysis. A number of software cuts have been applied to the parameterised pulses allowing the selection of single interactions within the fiducial volume of the detector to be made. These cuts are described in Refs. [9]. For each cut an energy dependent efficiency has been evaluated either from the data or from a combination of data and simulations.

An important cut that reduces significantly the fiducial volume of xenon, is the radial cut. This rejects the events that are reconstructed as being close to the PTFE walls. In reality, due to imperfect position reconstruction of the interaction points in the horizontal plane, a long tail of events assumed to be originated at the walls is reconstructed towards the centre of the detector. The majority of these events is believed to be caused by the alpha decay of radon progeny accumulated on the charged surfaces. To remove most of the 'wall' events the radial cut has been applied reducing the fiducial mass of xenon down to $7.2 \mathrm{~kg}$ (see Ref. [9] for more details).

Two xenon isotopes occurring naturally are sensitive to WIMP-nucleus spin-dependent interactions: ${ }^{129} \mathrm{Xe}$ and ${ }^{131} \mathrm{Xe}$. Other stable xenon isotopes are even-even (with even numbers of proton and neutrons) with very small coupling to WIMP spin. ${ }^{129} \mathrm{Xe}$ and ${ }^{131} \mathrm{Xe}$ are both odd-neutron isotopes and hence are sensitive mainly to WIMP-neutron interactions. Their relative abundances are $26.4 \%$ and $21.2 \%$, respectively. The effective exposure of these target isotopes to WIMPs is calculated as $X_{i}=M \times t \times C_{i} \times A \times \epsilon$, where $X_{i}$ is the exposure of the $i$ th isotope, $M=7.2 \mathrm{~kg}$ is the fiducial mass of the target, $t=31.2$ days is the live time of the run, $C_{i}$ is the relative abundance of the isotope, $A=0.5$ is the fraction of nuclear recoils in the acceptance window on S2/S1 vs S1 plane chosen for the analysis and $\epsilon$ is the overall energy-dependent efficiency of other cuts described above and in Ref. [9]. If we neglect the energy-dependent efficiency $\epsilon$ which increases from about $35 \%$ at $5 \mathrm{keV}$ to about $75 \%$ at $10 \mathrm{keV}$, the exposures for different xenon isotopes are $29.7 \mathrm{~kg} \times$ days for ${ }^{129} \mathrm{Xe}$ and $23.8 \mathrm{~kg} \times$ days for ${ }^{131} \mathrm{Xe}$. Cut efficiency $\epsilon$ further reduces the exposure making it also energy dependent.

The energy range of 5-20 keV (electron equivalent) has been chosen for the data analysis. Below $5 \mathrm{keV}$ the trigger efficiency is rather small (less than $40 \%$ ). Above $20 \mathrm{keV}$ the sensitivity of xenon target to WIMP interactions decreases significantly because of the rapidly falling form-factor. To avoid any bias, the selection of cuts and energy range for the final analysis was based on the results from calibration runs and from 'unblinded' $10 \%$ of data prior to 'opening the box' with the remaining $90 \%$ of data.

The analysis of data has revealed 29 events in the nuclear recoil acceptance box, the expected background rate due to electron recoils and 'wall' events being $28.6 \pm 4.3$ (see [9] for full description of the procedure to evaluate the expected background). Based on the previously published simulations
[12-15] we expect to have less than 1 nuclear recoil from neutron background in the detector for the aforementioned exposure.

Applying the procedure described by Feldman and Cousins [16] the 90\% CL upper limit on the number of nuclear recoils has been set as 10.4 using the ROOT software [17] and then converted into an upper limit on the WIMP-nucleon spinindependent cross-section [9]. The limit on the nuclear recoil rate can also be used to set a limit on spin-dependent interactions. This procedure is described below.

\section{Limits on spin-dependent cross-sections}

In the spin-independent case Majorana WIMP coupling to protons and neutrons is very similar, the coherence enhancement factor is proportional to $A^{2}$ and the cross-section on the nucleus does not depend on particular WIMP model parameters. For spin-dependent interactions the coupling to protons and neutrons is very much different and depends strongly on the WIMP model parameters. In the derivation of the spin-dependent limits we follow the procedure described in Ref. [18].

The WIMP-nucleus cross-section, $\sigma_{A}$, can be written as:

$\sigma_{A}=\frac{32}{\pi} G_{F}^{2} \mu_{A}^{2}\left(a_{p}\left\langle S_{p}\right\rangle+a_{n}\left\langle S_{n}\right\rangle\right)^{2} \frac{J+1}{J}$,

where $G_{F}$ is the Fermi weak coupling constant, $\mu_{A}$ is the WIMP-nucleus reduced mass, $a_{p, n}$ are the effective WIMPproton (neutron) couplings, $\left\langle S_{p, n}\right\rangle$ are the expectation values of the proton and neutron spins in the nucleus (or spin-factors) and $J$ is the nuclear spin. For a proton or a neutron as a target, Eq. (1) is transformed into the cross-section for WIMPproton (neutron) interactions with the proton (neutron) spins $\left\langle S_{p, n}\right\rangle=1 / 2$ and $J=1 / 2$. Eq. (1) does not correspond to the total WIMP-nucleus cross-section, but to that at zero momentum transfer. As in the case for spin-independent interactions, the cross-section for zero momentum transfer is usually presented for comparison with other results and model predictions. To evaluate the 'real' interaction cross-section and to compare it with the experimental data this has to be multiplied by the nuclear form-factor $F^{2}(q)$ which is a function of the momentum transfer, $q[19]$.

As in Ref. [18] we assume that the total WIMP-nucleus cross-section at zero momentum transfer is dominated by either WIMP-proton or WIMP-neutron interactions only, setting the 2 nd component equal to 0 . In this case the WIMP model dependent parameters are cancelled in the equation for the ratio of the cross-sections:

$\frac{\sigma_{p, n}}{\sigma_{A}}=\frac{3}{4} \frac{\mu_{p, n}^{2}}{\mu_{A}^{2}} \frac{1}{\left\langle S_{p, n}\right\rangle^{2}} \frac{J}{J+1}$,

which retains only the nuclear physics parameters. As $\sigma_{A}$ is measured in an experiment or a limit on $\sigma_{A}$ is set from the experimental data (assuming a particular form-factor) and nuclear physics parameters $\left\langle S_{p, n}\right\rangle$ can be calculated independently of the WIMP model, the WIMP-proton (neutron) cross-section can be evaluated in a (almost) model-independent way using 
Eq. (2). The only WIMP model dependence that remains is hidden in the form factor that appears to be different for different WIMP models. This dependence, however, is not significant for most isotopes and is comparable to the uncertainty in the nuclear physics model used.

For xenon as a target, additional complication comes from the existence of two isotopes with odd-neutron nuclei, ${ }^{129} \mathrm{Xe}$ and ${ }^{131} \mathrm{Xe}$. In this case the combined limit on the cross-section is calculated as [19]:

$\frac{1}{\sigma_{p, n}}=\sum_{A_{i}} \frac{1}{\sigma_{p, n}^{A_{i}}}$

where $\sigma_{p, n}^{A_{i}}$ are the WIMP-proton (neutron) limits set for crosssections on individual isotopes taking into account their fraction by weight, and $\sigma_{p, n}$ is the combined limit on the WIMP-proton (neutron) cross-section.

In further calculations we used the spherical isothermal dark matter halo model with the following parameters: $\rho_{\mathrm{dm}}=$ $0.3 \mathrm{GeV} \mathrm{cm}{ }^{-3}, v_{o}=220 \mathrm{~km} / \mathrm{s}, v_{\text {esc }}=600 \mathrm{~km} / \mathrm{s}$ and $v_{\text {Earth }}=$ $232 \mathrm{~km} / \mathrm{s}$. The form-factors were computed for the two xenon isotopes using the nuclear shell model calculations [20] with 'Bonn A' nucleon-nucleon potential. This is based on a comprehensive meson-exchange model for the nucleon-nucleon interaction in field theory developed by the Bonn group [21]. This model offers the most consistent approach to the nuclear manybody problem at low energies relevant to WIMP interactions. It includes all important diagrams with a total exchanged mass up to about the cutoff mass $(\sim 1 \mathrm{GeV})$. The various mesonexchange contributions in this mass range are introduced step by step proceeding from lowest-order to higher-order processes and from long range to short range. The model predictions agree well with the deuteron data and parameters derived from nucleon-nucleon scattering experiments [21].

The total form-factor can be written in the form:

$F^{2}(q)=\frac{S(q)}{S(0)}$,

where

$S(q)=a_{0}^{2} S_{00}(q)+a_{1}^{2} S_{11}(q)+a_{0} a_{1} S_{01}(q)$.

Here $a_{0}=a_{p}+a_{n}, a_{1}=a_{p}-a_{n}$ and $S_{00}, S_{11}, S_{01}$ are the isoscalar, isovector and interference contributions to the spin structure function $S(q)$, respectively. These contributions are determined by a nuclear model (independent of the WIMP type), with coefficients $a_{0}$ and $a_{1}$ related to the WIMP-proton and WIMP-neutron coupling constants $a_{p, n}$ (and hence WIMPtype dependent). The coupling coefficients depend also on the assumption about the quark spin fractions, i.e., the fractional contributions of different quark species to the nucleon spin. We used the WIMP-proton and WIMP-neutron coupling constants $a_{p}$ and $a_{n}$ with the quark spin fractions from Ref. [22]. Although the form-factor is normalised to the value at zero momentum transfer (Eq. (4)) that also includes the WIMP coupling coefficients, they do not cancel out completely, leaving some dependence on the WIMP particle model. The dependence on the WIMP model parameters for xenon isotopes is
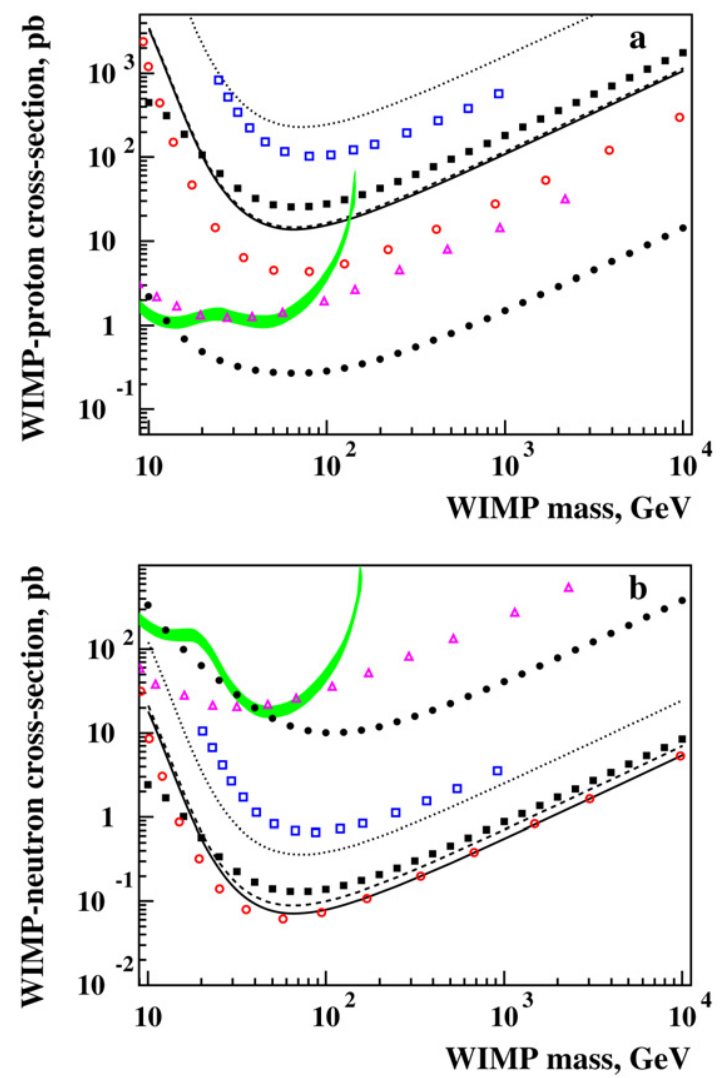

Fig. 1. 90\% CL upper limits on the WIMP-proton (a) and WIMP-neutron (b) spin-dependent cross-sections derived from ZEPLIN-II data. The limits from the two isotopes $\left({ }^{129} \mathrm{Xe}\right.$ - dashed curves, ${ }^{131} \mathrm{Xe}$ - dotted curves) and the combined limit (solid curves) are presented. The limits from some other experiments are also shown: NAIAD [2] (๑), ZEPLIN-I [4] (ם), CDMS [3] (O), EDELWEISS [5] $(\square)$ and PICASSO [23] $(\triangle)$ (the latter result coincides with that of the SIMPLE experiment [24]). The interpretation of the positive annual modulation signal observed by the DAMA experiment [1], in terms of the constraints on spin-dependent cross-sections reported by Savage et al. [25], is shown by the filled area.

not as weak as, for instance, for iodine and low-A isotopes. The form-factor for higgsino interactions, however, is the smallest among WIMP particle models, so the limit based on the present calculations with higgsino form-factor is conservative.

The spin-factors $\left\langle S_{p, n}\right\rangle$ for the two odd-neutron Xe isotopes have been calculated in Ref. [20] and also given in Ref. [18]. In the present analysis we used the values reported for the same nuclear model with Bonn A potential as for the form-factors.

Fig. 1 shows the 90\% CL limits on the WIMP-proton (a) and WIMP-neutron (b) spin-dependent cross-sections calculated using the ZEPLIN-II data and the procedure described above. Results from some other experiments are also shown. The ZEPLIN-II limits are also given in numerical form in Table 1 to allow more accurate comparison with other experiments. The minimum of the curve for the WIMP-neutron cross-section corresponds to $7 \times 10^{-2} \mathrm{pb}$. The limits are dominated by the contribution from the ${ }^{129} \mathrm{Xe}$ isotope. The ZEPLIN-II limits on WIMP-neutron cross-section are comparable to the currently best result obtained by the CDMS experiment [3]. Although the CDMS spin-independent limits are better than those from ZEPLIN-II, the spin-dependent WIMP-neutron cross-section 
Table 1

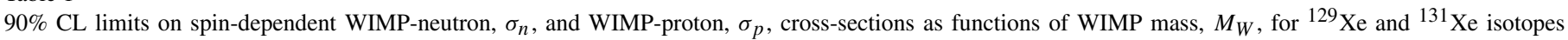
together with the combined limits from the ZEPLIN-II experiment

\begin{tabular}{|c|c|c|c|c|c|c|}
\hline$M_{W}(\mathrm{GeV})$ & $\sigma_{n}(\mathrm{pb}),{ }^{129} \mathrm{Xe}$ & $\sigma_{n}(\mathrm{pb}),{ }^{131} \mathrm{Xe}$ & $\sigma_{n}(\mathrm{pb})$ & $\sigma_{p}(\mathrm{pb}),{ }^{129} \mathrm{Xe}$ & $\sigma_{p}(\mathrm{pb}),{ }^{131} \mathrm{Xe}$ & $\sigma_{p}(\mathrm{pb})$ \\
\hline 10 & $2.11 \times 10^{1}$ & $1.21 \times 10^{2}$ & $1.79 \times 10^{1}$ & $3.46 \times 10^{3}$ & $7.71 \times 10^{4}$ & $3.31 \times 10^{3}$ \\
\hline 20 & $6.51 \times 10^{-1}$ & $3.47 \times 10^{0}$ & $5.48 \times 10^{-1}$ & $1.07 \times 10^{2}$ & $2.21 \times 10^{3}$ & $1.02 \times 10^{2}$ \\
\hline 40 & $1.14 \times 10^{-1}$ & $5.14 \times 10^{-1}$ & $9.31 \times 10^{-2}$ & $1.87 \times 10^{1}$ & $3.28 \times 10^{2}$ & $1.77 \times 10^{1}$ \\
\hline 63 & $8.89 \times 10^{-2}$ & $3.64 \times 10^{-1}$ & $7.15 \times 10^{-2}$ & $1.46 \times 10^{1}$ & $2.33 \times 10^{2}$ & $1.38 \times 10^{1}$ \\
\hline 100 & $1.00 \times 10^{-1}$ & $3.84 \times 10^{-1}$ & $7.94 \times 10^{-2}$ & $1.64 \times 10^{1}$ & $2.45 \times 10^{2}$ & $1.54 \times 10^{1}$ \\
\hline 200 & $1.61 \times 10^{-1}$ & $5.87 \times 10^{-1}$ & $1.26 \times 10^{-1}$ & $2.64 \times 10^{1}$ & $3.75 \times 10^{2}$ & $2.47 \times 10^{1}$ \\
\hline 400 & $2.96 \times 10^{-1}$ & $1.06 \times 10^{0}$ & $2.31 \times 10^{-1}$ & $4.86 \times 10^{1}$ & $6.76 \times 10^{2}$ & $4.54 \times 10^{1}$ \\
\hline 630 & $4.57 \times 10^{-1}$ & $1.63 \times 10^{0}$ & $3.57 \times 10^{-1}$ & $7.51 \times 10^{1}$ & $1.04 \times 10^{3}$ & $7.01 \times 10^{1}$ \\
\hline 1000 & $7.14 \times 10^{-1}$ & $2.53 \times 10^{0}$ & $5.57 \times 10^{-1}$ & $1.17 \times 10^{2}$ & $1.62 \times 10^{3}$ & $1.09 \times 10^{2}$ \\
\hline 10000 & $6.99 \times 10^{0}$ & $2.46 \times 10^{1}$ & $5.45 \times 10^{0}$ & $1.15 \times 10^{3}$ & $1.57 \times 10^{4}$ & $1.07 \times 10^{3}$ \\
\hline
\end{tabular}

limits presented here are very similar to those set by CDMS due to the higher fraction of odd-nucleon isotopes in xenon.

The uncertainties in the nuclear spin and form-factors used for the evaluation of limit are not negligible. Apart from the remaining dependence on the particle model, there is an uncertainty related to the nuclear model. Ressell and Dean [20] found a factor of 2 difference between their calculations of the spin structure functions for ${ }^{131} \mathrm{Xe}$ (higher values at zero momentum transfer) and earlier calculations by Engel [26]. The model used by Ressell and Dean [20] with Bonn A potential gives smaller (again by a factor of 2 approximately) spin structure functions than simple 'single particle' model. Slightly lower spin expectation value $S_{n}$ (by about $20 \%$ ) for ${ }^{129} \mathrm{Xe}$ was obtained with Nijmegen II potential [20] which results in a $20 \%$ higher limit on the cross-section. This makes the systematic uncertainty of the cross-section limit as large as a factor of 2 due to the nuclear model calculations. We stress, however, that the model used in the present analysis is based on the most recent and accurate calculations of nuclear spin and form-factors [20].

The predictions of different nuclear models for different nuclei are not strongly correlated in the sense that two models can predict, for instance, similar spin expectation values for one nucleus but very much different spins for another nucleus. Thus, possible future detection of WIMP interactions with several different target nuclei should help in reducing the uncertainties in nuclear physics models and, hence, in improving accuracy of the WIMP parameters' estimates.

The limits shown in Fig. 1 were obtained for a pure higgsino as a WIMP. Assuming pure photino or bino as a WIMP gives $35-40 \%$ better limit on the cross-section, whereas the limit for pure zino would be a factor of 3 lower. As precise composition of WIMPs is not known, the assumption of a pure higgsino leads to the most conservative limit.

To assess the significance of the spin-dependent limits less than $0.1 \mathrm{pb}$, it should be noted that a marginal dark matter candidate, the heavy Majorana neutrino, could have a spin-dependent cross-section of about $0.01 \mathrm{pb}$. However, a re-evaluation of likely supersymmetric candidates by Ellis [27] suggests spin-dependent cross-sections no greater than $10^{-4} \mathrm{pb}$.

Fig. 2 shows constraints on the WIMP-proton and WIMPneutron coupling coefficients $a_{p}$ and $a_{n}$ (for a WIMP mass of

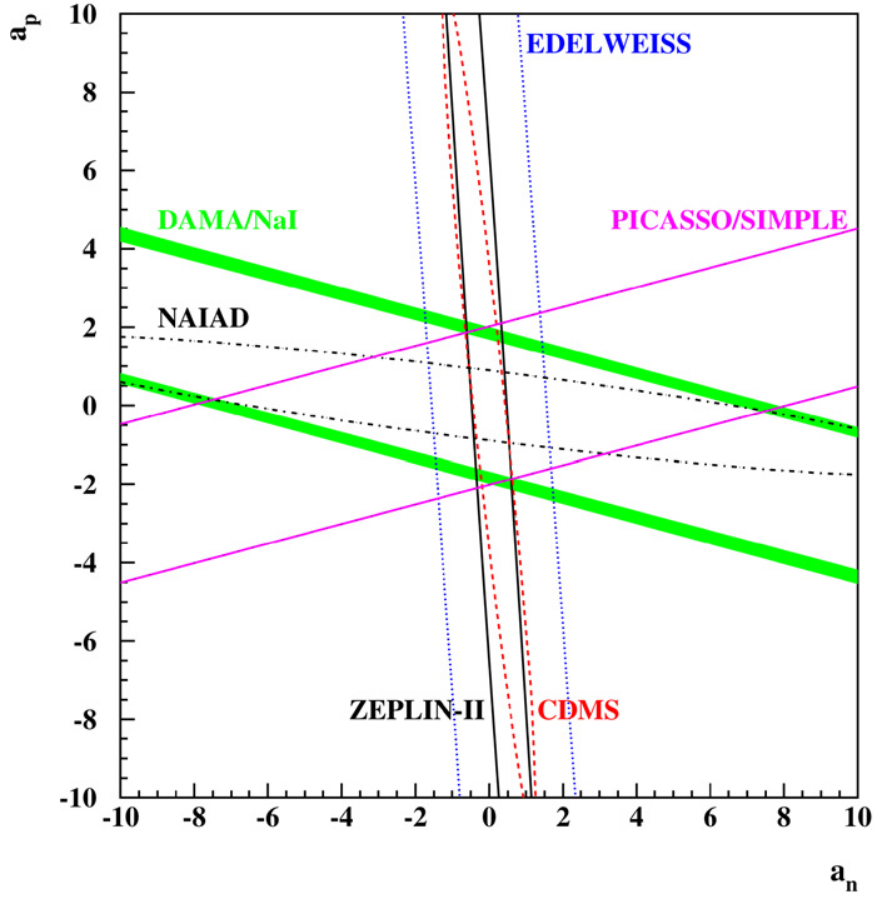

Fig. 2. Constraints on the WIMP-proton and WIMP-neutron coupling coefficients $a_{p}$ and $a_{n}$ (for a WIMP mass of $50 \mathrm{GeV}$ ) as derived from $90 \% \mathrm{CL}$ upper limits on the WIMP-proton and WIMP-neutron cross-sections from different experiments. The ZEPLIN-II results are shown by solid curves (almost vertical parallel lines). The region between the two lines is allowed by the ZEPLIN-II data. Other results are: CDMS (dashed curves) [3], EDELWEISS (dotted curves) [5], NAIAD (dashed-dotted curves, nearly horizontal) [2] and PICASSO/SIMPLE (solid curves, nearly horizontal) [23,24]. The allowed regions from the DAMA/NaI experiment [1] is shown by the filled area as calculated from the cross-section allowed regions reported in Ref. [25].

$50 \mathrm{GeV}$ ) as derived from the ZEPLIN-II data in comparison with other experiments. Here again we used the formalism described in Ref. [18] that allows conversion of the cross-section limits into the allowed regions on the $a_{p}-a_{n}$ plane using the equation:

$\sum_{A_{i}}\left(\frac{a_{p}}{\sqrt{\sigma_{p}^{A_{i}}}} \pm \frac{a_{n}}{\sqrt{\sigma_{n}^{A_{i}}}}\right)^{2}<\frac{\pi}{24 G_{F}^{2} \mu_{p}^{2}}$

where the small mass difference between the proton and the neutron is ignored. 
Strictly speaking the procedure is mathematically correct only if the form-factor used to evaluate the limits on crosssections $\sigma_{p, n}^{A_{i}}$ is independent of the WIMP model. In most cases (including ZEPLIN-II limit derivation in this Letter) the formfactor depends on the WIMP model, i.e. on the WIMP-proton and WIMP-neutron coupling coefficients $a_{p, n}$. In practice this means that the limits $\sigma_{p, n}^{A_{i}}$ depend on $a_{p, n}$. We used the formfactor for pure higgsino and in the derivation of the allowed regions on the $a_{p}-a_{n}$ plane we ignored the dependence of the form-factor, defined as $F^{2}(q)=S(q) / S(0)$, on the coupling coefficients. This remark concerns also other experiments $[2$, $5,23,24]$ for which similar procedure was used. We repeated the derivation of the allowed regions on the $a_{p}-a_{n}$ plane from the cross-section limits from these experiments and found them to be in good agreement with the original publications $[5,23,24]$. The allowed region from the CDMS experiment was copied from Ref. [3]. Our computation of the allowed region for CDMS gave slightly different result which implied that the CDMS Collaboration used another procedure for constraining coupling coefficients in Ref. [3]. The allowed region from the DAMA/NaI experiment [1] was calculated using the allowed regions for the cross-sections from Ref. [25].

One of the alternative methods of setting limits on the coupling constants was suggested in Ref. [25]. Despite its complexity, this method has an advantage of providing model independent constraints on the coupling coefficients by using the spin structure function $S(q)$ directly in the process of the data analysis, i.e. the coupling coefficients $a_{p, n}$ being free parameters in the fit to the data. Note, however, that for accurate comparison between different experiments, all data have to be analysed using the same method.

Fig. 2 shows that for $50 \mathrm{GeV}$ WIMP mass the DAMA allowed region (filled area on the figure) is excluded by the combination of other experiments. Savage et al. [25], however, found that the interpretation of the DAMA positive signal in terms of spin-dependent interactions is still compatible with other experiments at small WIMP masses $(5-13 \mathrm{GeV})$. NAIAD (sensitive mainly to $a_{p}$ ) and CDMS/ZEPLIN-II (sensitive mainly to $a_{n}$ ) provided so far the most stringent constraints on the WIMP-nucleon spin-dependent interactions.

\section{Conclusions}

The upper limits on the WIMP-proton and WIMP-neutron spin-dependent cross-sections have been set using the ZEPLINII data. The minimum of the curve for WIMP-neutron crosssection corresponds to $7 \times 10^{-2} \mathrm{pb}$. The limits on WIMPneutron cross-section are comparable to the currently best result obtained by the CDMS experiment.

\section{Acknowledgements}

This work has been funded by the UK Particle Physics And Astronomy Research Council (PPARC), the US Depart- ment of Energy (grant numbers DE-FG03-91ER40662 and DEFG03-95ER40917) and the US National Science Foundation (grant number PHY-0139065). We acknowledge support from the Central Laboratories for the Research Councils (CCLRC), the Engineering and Physical Sciences Research Council (EPSRC), the ILIAS integrating activity (Contract R113-CT-2004506222), the INTAS programme (grant number 04-78-6744) and the Research Corporation (grant number RA0350). We also acknowledge support from Fundação para a Ciência e Tecnologia (project POCI/FP/FNU/63446/2005), the Marie Curie International Reintegration Grant (grant number FP6-006651) and a PPARC PIPSS award (grant PP/D000742/1). We would like to gratefully acknowledge the strong support of Cleveland Potash Ltd., the owners of the Boulby mine, and J. Mulholland and L. Yeoman, the underground facility staff. We would like to thank the team from ITEP, Moscow, led by Dr. D. Akimov for their valuable contribution to the ZEPLIN programme. We acknowledge valuable advice from Prof. R. Cousins on the application and extension of the Feldman-Cousins tables.

\section{References}

[1] R. Bernabei, et al., Riv. Nuovo Cimento 26 (2003) 1.

[2] G.J. Alner, et al., UK Dark Matter Collaboration, Phys. Lett. B 616 (2005) 17.

[3] D.S. Akerib, et al., Phys. Rev. D 73 (2006) 011102(R).

[4] G.J. Alner, et al., UK Dark Matter Collaboration, in: N.J.C. Spooner, V.A. Kudryavtsev (Eds.), Proceedings of the 5th Intern. Workshop on the Identification of Dark Matter, Edinburgh, 6-10 September 2004, World Scientific, 2005, p. 218.

[5] A. Benoit, et al., Phys. Lett. B 616 (2005) 25.

[6] G.J. Alner, et al., New Astron. Rev. 49 (2005) 259.

[7] D. Cline, et al., Nucl. Phys. B (Proc. Suppl.) 124 (2002) 229.

[8] M. Robinson, et al., Nucl. Instrum. Methods Phys. Res. A 511 (2003) 347.

[9] G.J. Alner, et al., First limits on nuclear recoil signals in ZEPLIN-II: A two-phase xenon detector for dark matter detection, astro-ph/0701858, Astropart. Phys. (2007), doi:10.1016/j.astropartphys.2007.06.002, in press.

[10] G.J. Alner, et al., Nucl. Instrum. Methods Phys. Res. A; astro-ph/0703362, submitted for publication.

[11] G.J. Alner et al., in preparation.

[12] P.F. Smith, et al., Astropart. Phys. 22 (2004) 409.

[13] M.J. Carson, et al., Astropart. Phys. 21 (2004) 667.

[14] H.M. Araújo, V.A. Kudryavtsev, N.J.C. Spooner, T.J. Sumner, Nucl. Instrum. Methods Phys. Res. A 545 (2005) 398.

[15] C. Bungau, et al., Astropart. Phys. 23 (2005) 97.

[16] G.J. Feldman, R.D. Cousins, Phys. Rev. D 57 (1998) 3873.

[17] ROOT: An object oriented data analysis framework, http://root.cern.ch/.

[18] D.R. Tovey, et al., Phys. Lett. B 488 (2000) 17.

[19] J.D. Lewin, P.F. Smith, Astropart. Phys. 6 (1996) 87.

[20] M.T. Ressell, D.J. Dean, Phys. Rev. C 56 (1997) 535.

[21] R. Machleidt, K. Holinde, Ch. Elster, Phys. Rep. 149 (1987) 1.

[22] J. Ellis, M. Karliner, Phys. Lett. B 341 (1995) 397.

[23] M. Barnabè-Heider, et al., Phys. Lett. B 624 (2005) 186.

[24] T.A. Girard, et al., Phys. Lett. B 621 (2005) 233.

[25] C. Savage, P. Gondolo, K. Freese, Phys. Rev. D 70 (2004) 123513.

[26] J. Engel, Phys. Lett. B 264 (1991) 114.

[27] J.R. Ellis, Nucl. Phys. B (Proc. Suppl.) 101 (2000) 205. 